\title{
Radial oscillations of relativistic stars ${ }^{\star}$
}

\author{
K. D. Kokkotas ${ }^{1}$ and J. Ruoff ${ }^{1,2}$ \\ 1 Department of Physics, Aristotle University of Thessaloniki, Thessaloniki 54006, Greece \\ e-mail: kokkotas@astro.auth.gr; ruoff@astro.auth.gr \\ 2 Institut für Astronomie und Astrophysik, Universität Tübingen, Auf der Morgenstelle 10, 72076 Tübingen, \\ Germany
}

Received 1 September 2000 / Accepted 7 November 2000

\begin{abstract}
We present a new survey of the radial oscillation modes of neutron stars. This study complements and corrects earlier studies of radial oscillations. We present an extensive list of frequencies for the most common equations of state and some more recent ones. In order to check the accuracy, we use two different numerical schemes which yield the same results. The stimulation for this work comes from the need of the groups that evolve the full nonlinear Einstein equations to have reliable results from perturbation theory for comparison.
\end{abstract}

Key words. stars: neutron - stars: oscillations - equation of state - dense matter - gravitation

\section{Introduction}

As they are the simplest oscillation modes of neutron stars, radial modes have been the first under investigation, more than 35 years ago (Chandrasekhar 1964a, 1964b). More important, they can give information about the stability of the stellar model under consideration. Since radial oscillations do not couple to gravitational waves, the appropriate equations are quite simple, and it is relatively easy to numerically solve the eigenvalue problem that leads to the discrete set of oscillation frequencies of a neutron star. In the absence of any dissipative processes, the oscillation spectrum of a stable stellar model forms a complete set; it is therefore possible to describe any arbitrary periodic radial motion of a neutron star as a superposition of its various eigenmodes.

The radial modes of neutron stars have been thoroughly investigated by various authors mostly for zero temperature equations of state (EOS) (e.g. Harrison et al. 1965; Chanmugam 1977; Glass \& Lindblom 1983; Väth \& Chanmugam 1992 and references therein). But also protoneutron stars with a finite temperature EOS (Gondek et al. 1997) and strange stars were studied (Benvenuto \& Horvath 1991; Väth \& Chanmugam 1992; Gondek \& Zdunik 1999).

Send offprint requests to: K. D. Kokkotas

* Tables A.1 to A.20 are also available in electronic form at the CDS via anonymous ftp to cdsarc.u-strasbg.fr (130.79.128.5) or via http://cdsweb.u-strasbg.fr/cgi-bin/qcat?/A+A/366/565
The first exhaustive compilation of radial modes for various zero temperature EOS was presented by Glass \& Lindblom 1983 (hereafter GL). However, as was later pointed out by Väth \& Chanmugam 1992 (hereafter $\mathrm{VC})$, their numerical values for the oscillation frequencies seemed to be flawed although their equations were correct. $\mathrm{VC}$ computed the radial frequencies for 6 equations of state of dense matter and corroborated their own results using the argument (Harrison et al. 1965) that for the numerical code to be correct, it must yield a zero-frequency mode at exactly that central density for which the neutron star reaches its maximal mass. This is the point where the stellar model becomes unstable with respect to radial collapse if the central density is further increased. Yet, this is not the case for the results of GL, as was noticed by VC. However, the above mentioned test can be used only in the case when both in the stellar model and in the perturbation equations the equilibrium adiabatic index is used. In general, different adiabatic indices can be used depending on the physical conditions inside a star (Gondek et al. 1997). For example, if the slowness of weak interaction processes are taken into account, the regions of configurations stable with respect to radial perturbations extend beyond the central density of the star with the minimum mass (e.g. Chanmugam 1977) and of the star with maximum mass (Gourgoulhon et al. 1995).

In this paper, we repeat the numerical calculation of the radial oscillation modes of neutron stars for various zero temperature equations of state using the equilibrium adiabatic index. To verify the results, we use two 
different formulations of the equations together with two different numerical methods to solve the eigenvalue problem. We find that in all cases we obtain matching values for the eigenfrequencies. In addition we have verified that the codes yield zero frequency modes not only at the maxima but also at the minima of the mass curves.

We give corrected values for the equations of state used by GL, and we add some new equations of state. It is not clear to us what went wrong in their calculations, since for certain EOS our values agree with theirs (EOS C, E, O), for others they differ only slightly (EOS F, L, N), but for some EOS the discrepancy is quite large (EOS A, B, D, G, I).

Additionally we include six more recent equations of state: two models of Glendenning (1985), one of the model of Wiringa et al. (1988), the EOS MPA of Wu et al. (1991), and two EOS of Akmal et al. (1998). Finally, we include three more tables for polytropic equations of state with different polytropic indices. The form we use is given by

$p=\kappa \rho^{1+1 / n}$.

In particular, we present for the following values of $\kappa$ and $n:\left(n=1, \kappa=100 \mathrm{~km}^{2}\right),\left(n=0.8, \kappa=700 \mathrm{~km}^{2.5}\right),(n=$ $\left.0.5, \kappa=210^{5} \mathrm{~km}^{4}\right)$.

Another interesting feature is the occurrence of avoided mode crossings for realistic EOS. This phenomenon has been thoroughly studied by Gondek \& Zdunik (1999) for a realistic nucleon EOS and an EOS representing a strange star model. We find that it occurs for all considered realistic EOS, for some it is quite strongly pronounced, for others it is less obvious.

\section{Equations and numerical methods}

\subsection{The radial perturbation equations}

The static and spherically symmetric metric which describes an equilibrium stellar model is given by the following line element:

$\mathrm{d} s^{2}=-\mathrm{e}^{2 \nu} \mathrm{d} t^{2}+\mathrm{e}^{2 \lambda} \mathrm{d} r^{2}+r^{2}\left(\mathrm{~d} \theta^{2}+\sin ^{2} \theta \mathrm{d} \phi^{2}\right)$.

Together with the energy-momentum tensor for a perfect fluid

$T_{\mu \nu}=(\rho+p) u_{\mu} u_{\mu}+p g_{\mu \nu}$,

Einstein's field equations yield three independent ordinary differential equations for the four unknowns $\nu, \mu, \rho$, and $p$. To complete the set of equations, an equation of state

$p=p(\rho)$

must be supplemented. For a given central density, those equations then yield a unique stellar model with radius $R$ and mass $M$. Usually one introduces the mass function $m$ via

$\mathrm{e}^{-2 \lambda}=1-\frac{2 m(r)}{r}$

in order to replace the metric function $\lambda$.
To obtain the equations that govern the radial oscillations, both fluid and spacetime variables are perturbed in such a way that the spherical symmetry of the background body is not violated. If we define as $\delta r(r, t)$ the time dependent radial displacement of a fluid element located at the position $r$ in the unperturbed model and assume a harmonic time dependence

$\delta r(r, t)=X(r) \mathrm{e}^{i \omega t}$,

we obtain the following equation describing the radial oscillations

$$
\begin{aligned}
& C_{\mathrm{s}}^{2} X^{\prime \prime}+\left(\left(C_{\mathrm{s}}^{2}\right)^{\prime}-Z+4 \pi r \gamma p \mathrm{e}^{2 \lambda}-\nu^{\prime}\right) X^{\prime} \\
& +\left[2\left(\nu^{\prime}\right)^{2}+\frac{2 m}{r^{3}} \mathrm{e}^{2 \lambda}-Z^{\prime}-4 \pi(\rho+p) Z r \mathrm{e}^{2 \lambda}+\omega^{2} \mathrm{e}^{2 \lambda-2 \nu}\right] X \\
& =0
\end{aligned}
$$

where $C_{\mathrm{S}}$ is the sound speed, which is calculated from the unperturbed background for a specific equation of state

$C_{\mathrm{s}}^{2}=\frac{\mathrm{d} p}{\mathrm{~d} \rho}$,

and $\gamma$ is the adiabatic index, which, for adiabatic oscillations, is related to the sound speed through

$\gamma=\frac{\rho+p}{p} \frac{\mathrm{d} p}{\mathrm{~d} \rho}$.

Finally

$Z(r)=C_{\mathrm{s}}^{2}\left(\nu^{\prime}-\frac{2}{r}\right)$

The boundary condition at the center is that

$\delta r(r=0)=0$,

while at the surface, the Lagrangian variation of the pressure should vanish, i.e.

$\Delta p=0$.

This leads to the condition

$\gamma p \zeta(r)^{\prime}=0, \quad$ where $\zeta=r^{2} \mathrm{e}^{-\nu} X$.

Equation (7) together with the boundary conditions (11) and (13) form a self-adjoint boundary value problem for $\omega^{2}$.

As an alternative, the master Eq. (7) can be written in the variable $\zeta$ to yield Eq. (26.6) of Misner et al. (1973), which explicitly shows its self-adjoint nature:

$0=\frac{\mathrm{d}}{\mathrm{d} r}\left(P \frac{\mathrm{d} \zeta}{\mathrm{d} r}\right)+\left(Q+\omega^{2} W\right) \zeta$

with

$r^{2} W=(\rho+p) \mathrm{e}^{3 \lambda+\nu}$

$r^{2} P=\gamma p \mathrm{e}^{\lambda+3 \nu}$

$r^{2} Q=\mathrm{e}^{\lambda+3 \nu}(\rho+p)\left(\left(\nu^{\prime}\right)^{2}+4 \frac{\nu^{\prime}}{r}-8 \pi \mathrm{e}^{2 \lambda} p\right)$. 
At the origin, we have $\zeta(r=0)=0$, and at the surface, the boundary condition is also given by Eq. (13).

Since in both the general relativistic and in Newtonian theory, the oscillation problem is described by a SturmLiouville boundary value problem, the mathematical features that are known for the Newtonian problem (see Ledoux \& Walraven 1958) also apply to the general relativistic case, i.e. the frequency spectrum is discrete, there are $n$ nodes between the center and the surface of the eigenfunction of the $n$th mode, and the eigenfunctions are orthogonal.

Since $\omega$ is real for $\omega^{2}>0$, the solution is purely oscillatory. However for $\omega^{2}<0$, the frequency $\omega$ is imaginary, which corresponds to an exponentially growing solution. This means that for negative values of $\omega^{2}$, we have unstable radial oscillations. For neutron stars, it is the fundamental mode $\omega_{0}$ which becomes imaginary at central densities $\rho_{\mathrm{c}}$ larger than the critical density $\rho_{\text {crit }}$ for which the total stellar mass $M$ as a function of $\rho_{\mathrm{c}}$ is maximal. In this case, the star will ultimately collapse to a black hole. For $\rho_{\mathrm{c}}=\rho_{\text {crit }}$, the frequency of the fundamental mode $\omega_{0}$ must vanish. The higher modes become unstable for higher densities than for maximum mass models. For realistic equations of state, there are several regions in the mass-central-density curve, which are unstable. On the neutron star branch, there is another instability point on the low density side, where the star can become unstable with respect to explosion. This point limits the minimal mass of a neutron star.

\subsection{The numerical methods}

Since the radial perturbation problem is an old one, various methods have been used to estimate the radial mode frequencies for a given equation of state. A numerical integration scheme, which is similar to what we will describe here, has widely been used, while a Rayleigh-Ritz variational technique has also been used in the early times, see Bardeen et al. (1966) for details.

Given the discrepancies existing in the literature, we have derived the results via two different numerical methods.

The first method is called in numerical analysis the shooting method. In this case, one starts the integration for a trial value of $\omega^{2}$ and a given set of initial values of $X(r=0)$ and $X^{\prime}(r=0)$ which satisfy at the center the boundary condition (11) and integrates towards the surface. The discrete values of $\omega^{2}$ for which the boundary condition (13) is satisfied are the eigenfrequencies of the radial perturbations.

We will apply this method to Eq. (14), but we first transform it into two first order differential equations. By introducing

$\eta=P \zeta^{\prime}$,

we obtain

$\frac{\mathrm{d} \zeta}{\mathrm{d} r}=\frac{\eta}{P}$ $\frac{\mathrm{d} \eta}{\mathrm{d} r}=-\left(\omega^{2} W+Q\right) \zeta$

Through Taylor expansion, we find that close to the origin we have $\zeta(r)=\zeta_{0} r^{3}+\mathcal{O}\left(r^{5}\right)$ and $\eta(r)=\eta_{0}+\mathcal{O}\left(r^{2}\right)$. From Eq. (19) it then follows that the leading order coefficients are related by $3 \zeta_{0}=\eta_{0} / P(0)$. Choosing $\eta_{0}=1$, we obtain $\zeta_{0}=1 /(3 P(0))$, which gives us the initial values for the integration.

The second method is based on finite differencing of the radial perturbation Eq. (7) using second order accurate schemes for the spatial derivatives. The coefficients of the equation are calculated for a certain number of, say, $N$ grid points. In this way a matrix equation of the form

$\left(A-\omega_{n}^{2} \mathrm{I}\right) y=0, \quad 0 \leq n \leq N$

is constructed. $A$ is the tridiagonal matrix of the coefficients, I is the identity matrix, $\omega_{n}^{2}$ is the squared frequency of the $n$th mode, and $y$ is the vector with the unknown values of the eigenfunction of the specific mode at the $N$ grid points. The homogeneous linear Eq. (21) has a nontrivial solution only if the determinant of the coefficient matrix is equal to zero, i.e.

$\operatorname{det}\left|A-\omega_{n}^{2} \mathrm{I}\right|=0$.

This means that $\omega_{n}^{2}$ are the $N$ eigenvalues of the $N \times N$ matrix $A$. Their numerical evaluation has been achieved using the routines F01AKF and F02APF of the Nag library.

In this way one can calculate hundreds of radial eigenvalues for a specific stellar model in a single run. This method is more time consuming, but one avoids to search for each eigenvalue separately. In numerical analysis, this method is referred to as Numerov method.

Using both methods, we have calculated for each stellar model a large number of eigenvalues, though in the tables of the Appendix, we list only the three lowest ones. A further check of consistency is that for each EOS, the maximum mass model must yield zero frequency for the first mode. This is indeed the case as is it not for the results of GL.

\section{Results}

Although in principle we could compute the eigenfrequencies up to arbitrary precision, this would make no sense, since the overall accuracy of the frequencies is not limited by the machine precision, but by the number of tabulated values of the equation of state. For the construction of the stellar background model, one therefore has to interpolate between the given points. As it turns out, different interpolation scheme can yield different mode frequencies. Even though the bulk parameters of the stellar models are not very sensitive to the actual interpolation scheme, it is the profile of the sound speed, or equivalently, the adiabatic index which enters into the oscillation equations, and this quantity is highly sensitive to the interpolation scheme, especially in the regions where the EOS changes 
quite abruptly, such as, for instance, at the neutron drip point. Since this region lies in the low pressure regime and is therefore located close to the surface of the neutron star, it has a quite large influence on the modes because their amplitudes peak at the surface. From trying different interpolation schemes, such as linear logarithmic interpolation or spline interpolation, we find that the frequencies may vary up to about three per cent. We therefore tabulate our value with only two significant digits. Only for the polytropic equations of state, we include three significant digits, since in this case, the equation of state is analytic, and we do not have to rely on interpolation.

VC have given tabulated values for EOS D and EOS N. For EOS D, our results agree with theirs, however, for EOS $\mathrm{N}$ we find a quite significant discrepancy for the stellar parameters like mass and radius, especially in the high density regime. For instance, for $\rho_{\mathrm{c}}=210^{15} \mathrm{~g} / \mathrm{cm}^{3}$, they find a mass of $M=2.563 M_{\odot}$, whereas we obtain $M=2.621 M_{\odot}$. Since also GL find the former value, it seems that both GL and VC have used the tabulated version of the EOS N provided by Lindblom \& Detweiler (1983). If we also use this table, we, again, agree with VC, both in the stellar parameters and the radial oscillation frequencies. However, we have access to a table with a larger number of values (about twice a many in the density range from $10^{14} \mathrm{~g} / \mathrm{cm}^{3}$ to $\left.10^{16} \mathrm{~g} / \mathrm{cm}^{3}\right)$, which yields the latter value. In Table A.10, we give the frequencies obtained with the more refined EOS, which, especially for the fundamental mode, are quite different from the values of VC. These discrepancies show very drastically that the results are quite sensitive to the number of tabulated values of a given EOS.

In Fig. 1, we show the five radial modes as a function of the central density for the quite recent EOS APR1 (Akmal et al. 1998). It is clearly discernible that the fundamental mode becomes unstable at central densities above $2.3510^{15} \mathrm{~g} / \mathrm{cm}^{3}$. The instability point corresponds to a stellar model with the maximal allowed mass of $2.38 M_{\odot}$ and a radius of $10.77 \mathrm{~km}$.

Another prominent feature is the occurrence of a series of avoided crossings between the various modes. This peculiarity has been observed in previous calculations (Gondek et al. 1997), and has been extensively discussed by Gondek \& Zdunik (1999). It should be noted that those avoided crossings do not appear when a polytropic EOS is used (in this case, one also does not have the second instability point at the low density region), but it is a characteristic of realistic equations of state.

The phenomenon of avoided crossings is also known to appear for other types of oscillations. Depending on the stellar models, there can be avoided crossings between $g$ modes and $p$-modes. Furthermore, Anderson et al. (1996) have reported it to occur between the $f$-mode and the $w$-modes. Also in rotating stars a similar phenomenon shows up for the quasi-radial modes when their oscillation frequencies are plotted as a function of the rotational frequency (Clement 1986; Yoshida \& Eriguchi 1999).

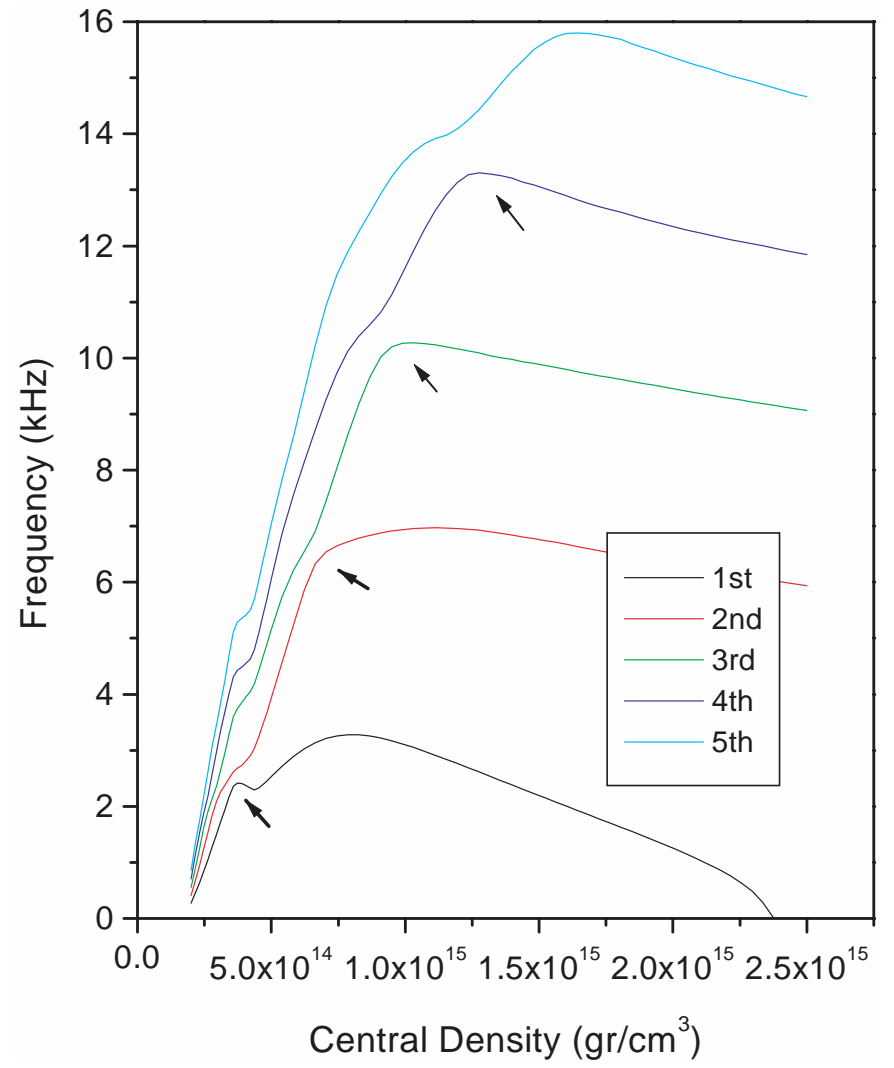

Fig. 1. We show the first five radial modes as a function of the central energy density. The frequency of the fundamental mode goes to zero at a density of about $2.3510^{15} \mathrm{~g} / \mathrm{cm}^{3}$, which indicates the onset of radial instability with respect to collapse to a black hole. The arrows indicate the avoided crossings between the different modes

All these cases have in common that there usually exist two or more families of modes, which arise from different physical origins. However, since they are described by a common set of equations, a particular frequency can only correspond to one single mode. Therefore, if the frequencies of two modes belonging to different families start to approach each other, they eventually have to repel each other before they come too close. This goes along with the modes exchanging their "family membership".

The radial oscillation modes, too, can be divided into two more or less independent families. According to Gondek \& Zdunik (1999), one family lives predominantly in the high density core of the neutron star and the other in the low density envelope. The two regions are divided by a "wall" in the adiabatic index, which results from the abrupt change in the stiffness of the matter at the neutron drip point (cf. Fig. 2 of Gondek \& Zdunik 1999). This wall effect is present for any realistic EOS, since it is associated with the neutron drip point, which belongs to the low pressure regime and is the same for all EOS.

In a model problem, Gondek \& Zdunik (1999) have decoupled both families, and in this case, both spectra show real crossings, when plotted on top over each other. When the coupling is brought back, the crossings vanish and the usual avoided crossing picture reemerges. 


\section{Summary}

We have presented updated results for radial oscillations of neutron stars using a quite exhaustive list of currently available equations of state, including some very recent ones. For most equations of state we significantly disagree with the values given by Glass \& Lindblom (1983). We have obtained our results by means of two different numerical methods, which agree up to arbitrary precision.

Furthermore, we have checked that our numerical codes yield zero frequency modes located exactly at both instability points, which are characterized by the local extrema in the mass-density curve. Here, we also obtain full agreement. The overall accuracy, however, is limited by the number of tabulated points for a given equation of state. Here, different numerical interpolation schemes may yield variations in the frequencies up to about three per cent.

Our results agree with the previous results of Väth \& Chanmugam (1992). However, we use a more complete table for the EOS N (Serot 1979), which significantly alters the values one obtains when the table provided by Lindblom \& Detweiler (1983) is used.

We have repeated the calculations for the equations of state already used by GL, and we have corrected their given values. In addition, we have included a large number of more recent equations of state. Since most of the present non-linear evolution codes use polytropic equations of state, we also have tabulated the mode frequencies for three different values of the polytropic index $n$.

Acknowledgements. J. R. was supported by the Deutsche Forschungsgemeinschaft through SFB 382 and the Marie Curie Fellowship No. HPMF-CT-1999-00364.

\section{References}

Akmal, A., Pandharipande, V. R, \& Ravenhall, D. G. 1998, Phys. Rev., C58, 1804

Andersson, N., Kojima, Y., \& Kokkotas, K. D. 1996, ApJ, 462, 855

Arponen, J. 1972, Nucl. Phys., A, 191, 257

Bardeen, J. M., Thorne, K. S., \& Meltzer, D. W. 1966, ApJ, 145,505

Bethe, H. A., \& Johnson, M. 1974, Nucl. Phys., A230, 1

Benvenuto, O. G., \& Horvath, J. E. 1991, MNRAS, 250, 679

Bowers, R. L., Gleeson, A. M., \& Pedigo, R. D. 1975, Phys. Rev., D12, 3043

Canuto, V., \& Chitre, S. M. 1974, Phys. Rev., D9, 1587

Chandrasekhar, S. 1964, Phys. Rev. Lett., 12, 114

Chandrasekhar, S. 1964, ApJ, 140, 417

Chanmugam, G. 1977, ApJ, 217, 799

Clement, M. J. 1986, ApJ, 301, 185

Cohen, J. M., Langer, W. D., Rosen, L. C., \& Cameron, A. G. W. 1970, Ap\&SS, 6, 228

Glass, E. N., \& Harpaz, A. 1983, MNRAS, 202, 159

Glass, E. N., \& Lindblom, L. 1983, ApJ, 53, 93, + Erratum, 1989, ApJ, 71, 173
Glendenning, N. K. 1985, ApJ, 293, 470

Gondek, D., Haensel, P., \& Zdunik, J. L. 1997, A\&A, 325, 217

Gondek, D., \& Zdunik, J. L. 1999, A\&A, 344, 117

Gourgoulhon, E., Haensel, P., \& Gondek, D. 1995, A\&A, 294, 747

Harrison, B. K., Thorne, K. S., Wakano, M., \& Wheeler, J. A., Gravitation Theory and Gravitational Collapse (Chicago: University of Chicago Press), 1965

Ledoux, P., \& Walraven, T. 1958, Hdb. d. Phys. (Berlin: Spinger-Verlag), 51, 458

Lindblom, L., \& Detweiler, S. L. 1983, ApJ, 53, 73

Meltzer, D. W., \& Thorne, K. S. 1966, ApJ, 145, 514

Misner, C. W., Thorne, K. S., \& Wheeler, J. A. 1973, Gravitation (Freeman \& Co., San Francisco)

Moszkowski, S. 1971, Phys. Rev., D9, 1613

Pandharipande, V. 1971, Nucl. Phys., A178, 123

Pandharipande, V., Pines, D., \& Smith, R. A. 1976, ApJ, 208, 550

Serot, B. D. 1979, Phys. Lett., 86B, 146; 87B, 403

Väth, H. M., Chanmugam, G. 1992, A\&A, 260, 250

Wiringa, R. B., Ficks, V., \& Fabrocini, A., Phys. Rev., C38, 1010

Wu, X., Müther, H., Soffel, M., Herold, H., \& Ruder, H. 1991, A\&A, 246, 411

Yoshida, S., \& Eriguchi, Y. [astro-ph/9908359]

\section{Appendix A: Results for various equations of state}

This appendix provides the numerical data for the radial mode frequencies of 17 realistic and 3 polytropic EOS. We present the data in the form of one table for each EOS. In each table we list the central density $\rho_{\mathrm{c}}$, the radius $R$, and the mass $M$ of the stellar model, and the frequencies $\nu_{n}=\omega_{n} /(2 \pi)$ of the first three radial modes. We also include one stellar model above the stability limit. For this case, we give the $e$-folding time in $m s$ for the fundamental mode, which is marked by an asterisk.

Table A.1. Data for the EOS A (Pandharipande 1971)

\begin{tabular}{cccccc}
\hline$\rho_{\mathrm{c}} 10^{15}$ & $R$ & $M$ & $\nu_{0}$ & $\nu_{1}$ & $\begin{array}{c}\nu_{2} \\
\mathrm{~g} / \mathrm{cm}^{3}\end{array}$ \\
\hline $\mathrm{km}$ & $M_{\odot}$ & $\mathrm{kHz}$ & $\mathrm{kHz}$ & $\mathrm{kHz}$ \\
\hline 4.200 & 8.335 & 1.654 & $0.34^{*}$ & 7.55 & 11.91 \\
4.100 & 8.373 & 1.654 & 0.28 & 7.58 & 11.95 \\
3.980 & 8.419 & 1.654 & 0.66 & 7.63 & 12.00 \\
3.000 & 8.874 & 1.621 & 1.97 & 7.98 & 12.33 \\
2.344 & 9.256 & 1.536 & 2.62 & 8.29 & 12.27 \\
1.995 & 9.479 & 1.447 & 2.94 & 8.46 & 11.88 \\
1.698 & 9.667 & 1.329 & 3.23 & 8.57 & 11.31 \\
1.259 & 9.890 & 1.050 & 3.67 & 8.04 & 10.67 \\
\hline
\end{tabular}


Table A.2. Data for the EOS B (Pandharipande 1971)

\begin{tabular}{cccccc}
\hline $\begin{array}{c}\rho_{\mathrm{c}} 10^{15} \\
\mathrm{~g} / \mathrm{cm}^{3}\end{array}$ & $\begin{array}{c}R \\
\mathrm{~km}\end{array}$ & $\begin{array}{c}M \\
M_{\odot}\end{array}$ & $\begin{array}{c}\nu_{0} \\
\mathrm{kHz}\end{array}$ & $\begin{array}{c}\nu_{1} \\
\mathrm{kHz}\end{array}$ & $\begin{array}{c}\nu_{2} \\
\mathrm{kHz}\end{array}$ \\
\hline 6.100 & 7.024 & 1.413 & $0.32^{*}$ & 8.75 & 13.18 \\
6.000 & 7.048 & 1.413 & 0.25 & 8.76 & 13.20 \\
5.900 & 7.072 & 1.413 & 0.61 & 8.78 & 13.21 \\
5.500 & 7.175 & 1.411 & 1.30 & 8.86 & 13.32 \\
5.012 & 7.316 & 1.404 & 1.82 & 8.91 & 13.45 \\
3.981 & 7.686 & 1.361 & 2.69 & 8.94 & 13.71 \\
3.388 & 7.953 & 1.304 & 3.10 & 8.86 & 13.71 \\
3.000 & 8.145 & 1.247 & 3.34 & 8.81 & 13.54 \\
1.995 & 8.766 & 0.971 & 3.58 & 8.65 & 11.28 \\
\hline
\end{tabular}

Table A.3. Data for the EOS C (Bethe \& Johnson 1974, model I)

\begin{tabular}{cccccc}
\hline $\begin{array}{c}\rho_{\mathrm{c}} 10^{15} \\
\mathrm{~g} / \mathrm{cm}^{3}\end{array}$ & $\begin{array}{c}R \\
\mathrm{~km}\end{array}$ & $\begin{array}{c}M \\
M_{\odot}\end{array}$ & $\begin{array}{c}\nu_{0} \\
\mathrm{kHz}\end{array}$ & $\begin{array}{c}\nu_{1} \\
\mathrm{kHz}\end{array}$ & $\begin{array}{c}\nu_{2} \\
\mathrm{kHz}\end{array}$ \\
\hline 3.100 & 9.884 & 1.852 & $0.39^{*}$ & 6.23 & 9.55 \\
3.000 & 9.952 & 1.852 & 0.30 & 6.23 & 9.54 \\
2.800 & 10.095 & 1.850 & 0.79 & 6.25 & 9.54 \\
2.500 & 10.326 & 1.840 & 1.24 & 6.28 & 9.54 \\
1.995 & 10.779 & 1.790 & 1.80 & 6.34 & 9.60 \\
1.778 & 11.010 & 1.746 & 2.02 & 6.34 & 9.58 \\
1.413 & 11.443 & 1.619 & 2.36 & 6.33 & 9.47 \\
1.122 & 11.834 & 1.436 & 2.56 & 6.25 & 9.23 \\
1.000 & 12.018 & 1.323 & 2.59 & 6.13 & 8.90 \\
\hline
\end{tabular}

Table A.4. Data for the EOS D (Bethe \& Johnson 1974, model V)

\begin{tabular}{cccccc}
\hline $\begin{array}{c}\rho_{\mathrm{c}} 10^{15} \\
\mathrm{~g} / \mathrm{cm}^{3}\end{array}$ & $\begin{array}{c}R \\
\mathrm{~km}\end{array}$ & $\begin{array}{c}M \\
M_{\odot}\end{array}$ & $\begin{array}{c}\nu_{0} \\
\mathrm{kHz}\end{array}$ & $\begin{array}{c}\nu_{1} \\
\mathrm{kHz}\end{array}$ & $\begin{array}{c}\nu_{2} \\
\mathrm{kHz}\end{array}$ \\
\hline 3.370 & 9.360 & 1.651 & $0.71^{*}$ & 6.99 & 10.27 \\
3.300 & 9.403 & 1.651 & 0.30 & 6.99 & 10.27 \\
3.000 & 9.598 & 1.648 & 0.84 & 6.96 & 10.32 \\
2.512 & 9.944 & 1.631 & 1.36 & 6.81 & 10.46 \\
1.778 & 10.447 & 1.547 & 2.43 & 6.71 & 9.88 \\
1.413 & 10.678 & 1.424 & 2.96 & 7.25 & 10.37 \\
1.122 & 10.965 & 1.186 & 3.09 & 6.93 & 9.71 \\
\hline
\end{tabular}

Table A.5. Data for the EOS E (Moszkowski 1974)

\begin{tabular}{cccccc}
\hline $\begin{array}{c}\rho_{\mathrm{c}} 10^{15} \\
\mathrm{~g} / \mathrm{cm}^{3}\end{array}$ & $\begin{array}{c}R \\
\mathrm{~km}\end{array}$ & $\begin{array}{c}M \\
M_{\odot}\end{array}$ & $\begin{array}{c}\nu_{0} \\
\mathrm{kHz}\end{array}$ & $\begin{array}{c}\nu_{1} \\
\mathrm{kHz}\end{array}$ & $\begin{array}{c}\nu_{2} \\
\mathrm{kHz}\end{array}$ \\
\hline 3.000 & 9.061 & 1.726 & 1.78 & 7.62 & 11.56 \\
2.818 & 9.171 & 1.711 & 1.98 & 7.68 & 11.60 \\
2.239 & 9.562 & 1.624 & 2.58 & 7.84 & 11.65 \\
1.778 & 9.915 & 1.474 & 3.02 & 7.90 & 11.44 \\
1.585 & 10.068 & 1.376 & 3.18 & 7.86 & 11.19 \\
1.259 & 10.314 & 1.144 & 3.39 & 7.59 & 10.36 \\
\hline
\end{tabular}

Table A.6. Data for the EOS F (Arponen 1972)

\begin{tabular}{cccccc}
\hline $\begin{array}{c}\rho_{\mathrm{c}} 10^{15} \\
\mathrm{~g} / \mathrm{cm}^{3}\end{array}$ & $\begin{array}{c}R \\
\mathrm{~km}\end{array}$ & $\begin{array}{c}M \\
M_{\odot}\end{array}$ & $\begin{array}{c}\nu_{0} \\
\mathrm{kHz}\end{array}$ & $\begin{array}{c}\nu_{1} \\
\mathrm{kHz}\end{array}$ & $\begin{array}{c}\nu_{2} \\
\mathrm{kHz}\end{array}$ \\
\hline 5.200 & 7.881 & 1.463 & $0.40^{*}$ & 7.40 & 11.88 \\
5.100 & 7.922 & 1.463 & 0.20 & 7.41 & 11.86 \\
5.012 & 7.961 & 1.463 & 0.46 & 7.42 & 11.83 \\
4.500 & 8.204 & 1.459 & 1.09 & 7.48 & 11.66 \\
3.981 & 8.490 & 1.449 & 1.42 & 7.54 & 11.49 \\
3.162 & 9.088 & 1.412 & 1.63 & 7.40 & 11.15 \\
2.239 & 9.923 & 1.333 & 1.84 & 6.76 & 10.23 \\
1.585 & 10.465 & 1.222 & 2.36 & 6.62 & 9.74 \\
1.122 & 10.889 & 1.032 & 2.75 & 6.57 & 9.03 \\
\hline
\end{tabular}

Table A.7. Data for the EOS G (Canuto \& Chitre 1974))

\begin{tabular}{cccccc}
\hline $\begin{array}{c}\rho_{\mathrm{c}} 10^{15} \\
\mathrm{~g} / \mathrm{cm}^{3}\end{array}$ & $\begin{array}{c}R \\
\mathrm{~km}\end{array}$ & $\begin{array}{c}M \\
M_{\odot}\end{array}$ & $\begin{array}{c}\nu_{0} \\
\mathrm{kHz}\end{array}$ & $\begin{array}{c}\nu_{1} \\
\mathrm{kHz}\end{array}$ & $\begin{array}{c}\nu_{2} \\
\mathrm{kHz}\end{array}$ \\
\hline 6.300 & 6.945 & 1.357 & $0.70^{*}$ & 8.77 & 13.54 \\
6.200 & 6.970 & 1.357 & 0.48 & 8.77 & 13.54 \\
6.100 & 6.996 & 1.357 & 0.72 & 8.77 & 13.54 \\
5.800 & 7.075 & 1.356 & 1.18 & 8.79 & 13.53 \\
5.500 & 7.159 & 1.353 & 1.53 & 8.81 & 13.52 \\
5.000 & 7.308 & 1.344 & 2.00 & 8.87 & 13.51 \\
4.503 & 7.472 & 1.327 & 2.40 & 8.95 & 13.53 \\
3.498 & 7.899 & 1.253 & 2.98 & 8.97 & 13.61 \\
2.631 & 8.399 & 1.114 & 3.25 & 8.48 & 12.54 \\
2.376 & 8.557 & 1.057 & 3.35 & 8.34 & 11.86 \\
\hline
\end{tabular}

Table A.8. Data for the EOS I (Cohen et al. 1970)

\begin{tabular}{cccccc}
\hline $\begin{array}{c}\rho_{\mathrm{c}} 10^{15} \\
\mathrm{~g} / \mathrm{cm}^{3}\end{array}$ & $\begin{array}{c}R \\
\mathrm{~km}\end{array}$ & $\begin{array}{c}M \\
M_{\odot}\end{array}$ & $\begin{array}{c}\nu_{0} \\
\mathrm{kHz}\end{array}$ & $\begin{array}{c}\nu_{1} \\
\mathrm{kHz}\end{array}$ & $\begin{array}{c}\nu_{2} \\
\mathrm{kHz}\end{array}$ \\
\hline 2.100 & 11.795 & 2.446 & $0.33^{*}$ & 5.27 & 8.15 \\
2.000 & 11.900 & 2.447 & 0.24 & 5.32 & 8.23 \\
1.800 & 12.161 & 2.441 & 0.84 & 5.40 & 8.29 \\
1.585 & 12.470 & 2.418 & 1.22 & 5.50 & 8.41 \\
1.259 & 13.025 & 2.324 & 1.69 & 5.60 & 8.52 \\
1.000 & 13.499 & 2.154 & 2.05 & 5.74 & 8.61 \\
0.794 & 13.883 & 1.883 & 2.31 & 5.77 & 8.51 \\
0.631 & 14.127 & 1.561 & 2.46 & 5.67 & 7.95 \\
\hline
\end{tabular}

Table A.9. Data for the EOS L (Pandharipande et al. 1976)

\begin{tabular}{cccccc}
\hline $\begin{array}{c}\rho_{\mathrm{c}} 10^{15} \\
\mathrm{~g} / \mathrm{cm}^{3}\end{array}$ & $\begin{array}{c}R \\
\mathrm{~km}\end{array}$ & $\begin{array}{c}M \\
M_{\odot}\end{array}$ & $\begin{array}{c}\nu_{0} \\
\mathrm{kHz}\end{array}$ & $\begin{array}{c}\nu_{1} \\
\mathrm{kHz}\end{array}$ & $\begin{array}{c}\nu_{2} \\
\mathrm{kHz}\end{array}$ \\
\hline 1.500 & 13.618 & 2.662 & $0.68^{*}$ & 4.66 & 7.39 \\
1.400 & 13.747 & 2.660 & 0.56 & 4.70 & 7.47 \\
1.259 & 13.936 & 2.649 & 0.97 & 4.79 & 7.58 \\
1.150 & 14.087 & 2.630 & 1.25 & 4.92 & 7.69 \\
1.000 & 14.299 & 2.579 & 1.59 & 5.21 & 8.05 \\
0.794 & 14.681 & 2.391 & 2.03 & 5.66 & 8.35 \\
0.631 & 14.989 & 2.044 & 2.27 & 5.71 & 8.20 \\
0.600 & 15.025 & 1.959 & 2.32 & 5.69 & 8.15 \\
0.500 & 15.056 & 1.636 & 2.53 & 5.58 & 7.52 \\
0.398 & 14.889 & 1.214 & 2.77 & 5.47 & 6.09 \\
\hline
\end{tabular}


Table A.10. Data for the EOS N (Serot 1979)

\begin{tabular}{cccccc}
\hline $\begin{array}{c}\rho_{\mathrm{c}} 10^{15} \\
\mathrm{~g} / \mathrm{cm}^{3}\end{array}$ & $\begin{array}{c}R \\
\mathrm{~km}\end{array}$ & $\begin{array}{c}M \\
M_{\odot}\end{array}$ & $\begin{array}{c}\nu_{0} \\
\mathrm{kHz}\end{array}$ & $\begin{array}{c}\nu_{1} \\
\mathrm{kHz}\end{array}$ & $\begin{array}{c}\nu_{2} \\
\mathrm{kHz}\end{array}$ \\
\hline 1.700 & 12.740 & 2.634 & $0.47^{*}$ & 5.10 & 7.97 \\
1.600 & 12.852 & 2.633 & 0.49 & 5.19 & 8.09 \\
1.400 & 13.107 & 2.619 & 1.03 & 5.36 & 8.30 \\
1.200 & 13.385 & 2.575 & 1.47 & 5.62 & 8.57 \\
1.000 & 13.686 & 2.468 & 1.90 & 5.90 & 8.88 \\
0.800 & 13.951 & 2.233 & 2.39 & 6.27 & 9.19 \\
0.600 & 13.980 & 1.729 & 2.96 & 6.61 & 8.79 \\
0.500 & 13.757 & 1.313 & 3.20 & 6.38 & 7.56 \\
0.400 & 13.349 & 0.836 & 3.26 & 5.02 & 5.68 \\
\hline
\end{tabular}

Table A.11. Data for the EOS O (Bowers et al. 1975)

\begin{tabular}{cccccc}
\hline $\begin{array}{c}\rho_{\mathrm{c}} 10^{15} \\
\mathrm{~g} / \mathrm{cm}^{3}\end{array}$ & $\begin{array}{c}R \\
\mathrm{~km}\end{array}$ & $\begin{array}{c}M \\
M_{\odot}\end{array}$ & $\begin{array}{c}\nu_{0} \\
\mathrm{kHz}\end{array}$ & $\begin{array}{c}\nu_{1} \\
\mathrm{kHz}\end{array}$ & $\begin{array}{c}\nu_{2} \\
\mathrm{kHz}\end{array}$ \\
\hline 2.100 & 11.502 & 2.379 & $0.55^{*}$ & 5.56 & 8.64 \\
2.000 & 11.587 & 2.378 & 0.53 & 5.63 & 8.72 \\
1.800 & 11.765 & 2.370 & 1.05 & 5.80 & 8.99 \\
1.600 & 11.974 & 2.346 & 1.43 & 5.94 & 9.23 \\
1.400 & 12.201 & 2.296 & 1.81 & 6.14 & 9.51 \\
1.200 & 12.442 & 2.199 & 2.18 & 6.38 & 9.93 \\
1.000 & 12.672 & 2.019 & 2.56 & 6.75 & 10.21 \\
0.800 & 12.832 & 1.682 & 2.86 & 7.09 & 9.43 \\
0.600 & 12.760 & 1.173 & 3.09 & 6.58 & 7.92 \\
\hline
\end{tabular}

Table A.12. Data for the EOS $\mathrm{G}_{240}$ (Glendenning 1985)

\begin{tabular}{cccccc}
\hline $\begin{array}{c}\rho_{\mathrm{c}} 10^{15} \\
\mathrm{~g} / \mathrm{cm}^{3}\end{array}$ & $\begin{array}{c}R \\
\mathrm{~km}\end{array}$ & $\begin{array}{c}M \\
M_{\odot}\end{array}$ & $\begin{array}{c}\nu_{0} \\
\mathrm{kHz}\end{array}$ & $\begin{array}{c}\nu_{1} \\
\mathrm{kHz}\end{array}$ & $\begin{array}{c}\nu_{2} \\
\mathrm{kHz}\end{array}$ \\
\hline 2.600 & 10.850 & 1.553 & $0.66^{*}$ & 5.39 & 8.59 \\
2.500 & 10.928 & 1.553 & 0.33 & 5.40 & 8.58 \\
2.200 & 11.209 & 1.549 & 0.77 & 5.42 & 8.48 \\
1.800 & 11.647 & 1.529 & 1.11 & 5.43 & 8.37 \\
1.400 & 12.200 & 1.481 & 1.37 & 5.34 & 8.10 \\
1.000 & 12.798 & 1.374 & 1.70 & 5.28 & 7.98 \\
0.800 & 13.110 & 1.267 & 1.91 & 5.17 & 7.96 \\
0.600 & 13.351 & 1.095 & 2.27 & 5.27 & 6.86 \\
0.400 & 13.482 & 0.711 & 2.36 & 4.54 & 5.23 \\
\hline
\end{tabular}

Table A.13. Data for the EOS G300 (Glendenning 1985)

\begin{tabular}{cccccc}
\hline $\begin{array}{c}\rho_{\mathrm{c}} 10^{15} \\
\mathrm{~g} / \mathrm{cm}^{3}\end{array}$ & $\begin{array}{c}R \\
\mathrm{~km}\end{array}$ & $\begin{array}{c}M \\
M_{\odot}\end{array}$ & $\begin{array}{c}\nu_{0} \\
\mathrm{kHz}\end{array}$ & $\begin{array}{c}\nu_{1} \\
\mathrm{kHz}\end{array}$ & $\begin{array}{c}\nu_{2} \\
\mathrm{kHz}\end{array}$ \\
\hline 2.200 & 11.687 & 1.788 & $0.33^{*}$ & 5.02 & 8.06 \\
2.100 & 11.762 & 1.788 & 0.12 & 5.08 & 8.10 \\
2.000 & 11.842 & 1.787 & 0.51 & 5.15 & 8.15 \\
1.800 & 12.027 & 1.782 & 0.86 & 5.25 & 8.23 \\
1.400 & 12.542 & 1.742 & 1.28 & 5.37 & 8.22 \\
1.000 & 13.182 & 1.624 & 1.66 & 5.36 & 7.99 \\
0.800 & 13.482 & 1.506 & 1.92 & 5.42 & 8.12 \\
0.600 & 13.718 & 1.286 & 2.27 & 5.33 & 7.50 \\
0.500 & 13.733 & 1.119 & 2.54 & 5.61 & 6.60 \\
0.400 & 13.630 & 0.825 & 2.58 & 5.04 & 5.59 \\
\hline
\end{tabular}

Table A.14. Data for the EOS WFF (Wiringa et al. 1988)

\begin{tabular}{cccccc}
\hline $\begin{array}{c}\rho_{\mathrm{c}} 10^{15} \\
\mathrm{~g} / \mathrm{cm}^{3}\end{array}$ & $\begin{array}{c}R \\
\mathrm{~km}\end{array}$ & $\begin{array}{c}M \\
M_{\odot}\end{array}$ & $\begin{array}{c}\nu_{0} \\
\mathrm{kHz}\end{array}$ & $\begin{array}{c}\nu_{1} \\
\mathrm{kHz}\end{array}$ & $\begin{array}{c}\nu_{2} \\
\mathrm{kHz}\end{array}$ \\
\hline 3.200 & 9.510 & 1.840 & $0.47^{*}$ & 6.66 & 10.38 \\
3.100 & 9.558 & 1.840 & 0.42 & 6.70 & 10.44 \\
3.000 & 9.612 & 1.840 & 0.69 & 6.73 & 10.45 \\
2.800 & 9.729 & 1.836 & 1.08 & 6.80 & 10.49 \\
2.600 & 9.850 & 1.828 & 1.38 & 6.90 & 10.55 \\
2.000 & 10.278 & 1.759 & 2.13 & 7.20 & 10.89 \\
1.800 & 10.441 & 1.710 & 2.37 & 7.30 & 10.99 \\
1.400 & 10.774 & 1.538 & 2.87 & 7.47 & 11.12 \\
1.200 & 10.925 & 1.389 & 3.12 & 7.52 & 10.78 \\
1.000 & 11.038 & 1.178 & 3.34 & 7.54 & 9.42 \\
0.900 & 11.075 & 1.044 & 3.41 & 7.46 & 8.51 \\
0.800 & 11.104 & 0.889 & 3.42 & 6.99 & 7.68 \\
\hline
\end{tabular}

Table A.15. Data for the EOS MPA (Wu et al. 1991)

\begin{tabular}{cccccc}
\hline $\begin{array}{c}\rho_{\mathrm{c}} 10^{15} \\
\mathrm{~g} / \mathrm{cm}^{3}\end{array}$ & $\begin{array}{c}R \\
\mathrm{~km}\end{array}$ & $\begin{array}{c}M \\
M_{\odot}\end{array}$ & $\begin{array}{c}\nu_{0} \\
\mathrm{kHz}\end{array}$ & $\begin{array}{c}\nu_{1} \\
\mathrm{kHz}\end{array}$ & $\begin{array}{c}\nu_{2} \\
\mathrm{kHz}\end{array}$ \\
\hline 4.800 & 7.899 & 1.560 & $0.39^{*}$ & 7.82 & 11.96 \\
4.700 & 7.930 & 1.560 & 0.37 & 7.85 & 11.99 \\
4.600 & 7.963 & 1.559 & 0.67 & 7.87 & 12.02 \\
4.500 & 7.996 & 1.559 & 0.87 & 7.90 & 12.06 \\
4.400 & 8.030 & 1.558 & 1.04 & 7.92 & 12.10 \\
4.200 & 8.104 & 1.556 & 1.31 & 7.96 & 12.14 \\
4.000 & 8.186 & 1.551 & 1.55 & 7.99 & 12.16 \\
3.500 & 8.407 & 1.531 & 2.07 & 8.08 & 12.26 \\
3.000 & 8.669 & 1.489 & 2.51 & 8.13 & 12.24 \\
2.500 & 8.973 & 1.410 & 2.90 & 8.16 & 12.10 \\
2.000 & 9.328 & 1.269 & 3.19 & 8.08 & 11.61 \\
1.500 & 9.747 & 1.033 & 3.29 & 7.58 & 10.48 \\
1.200 & 10.031 & 0.844 & 3.27 & 6.95 & 9.00 \\
1.000 & 10.251 & 0.698 & 3.22 & 6.36 & 7.47 \\
\hline
\end{tabular}

Table A.16. Data for the EOS APR1 (Akmal et al. 1998)

\begin{tabular}{cccccc}
\hline $\begin{array}{c}\rho_{\mathrm{c}} 10^{15} \\
\mathrm{~g} / \mathrm{cm}^{3}\end{array}$ & $\begin{array}{c}R \\
\mathrm{~km}\end{array}$ & $\begin{array}{c}M \\
M_{\odot}\end{array}$ & $\begin{array}{c}\nu_{0} \\
\mathrm{kHz}\end{array}$ & $\begin{array}{c}\nu_{1} \\
\mathrm{kHz}\end{array}$ & $\begin{array}{c}\nu_{2} \\
\mathrm{kHz}\end{array}$ \\
\hline 2.400 & 10.746 & 2.379 & $0.40^{*}$ & 6.01 & 9.14 \\
2.300 & 10.822 & 2.379 & 0.47 & 6.08 & 9.21 \\
2.200 & 10.904 & 2.377 & 0.80 & 6.16 & 9.29 \\
2.100 & 10.990 & 2.373 & 1.04 & 6.24 & 9.37 \\
2.000 & 11.080 & 2.366 & 1.25 & 6.32 & 9.45 \\
1.800 & 11.277 & 2.340 & 1.64 & 6.50 & 9.62 \\
1.500 & 11.611 & 2.250 & 2.19 & 6.76 & 9.89 \\
1.200 & 11.966 & 2.040 & 2.75 & 6.96 & 10.16 \\
1.000 & 12.171 & 1.774 & 3.10 & 6.94 & 10.27 \\
0.800 & 12.294 & 1.365 & 3.28 & 6.75 & 8.82 \\
0.700 & 12.336 & 1.109 & 3.21 & 6.53 & 7.39 \\
0.600 & 12.435 & 0.841 & 2.95 & 5.52 & 6.37 \\
\hline
\end{tabular}


Table A.17. Data for the EOS APR2 (Akmal et al. 1998)

\begin{tabular}{cccccc}
\hline $\begin{array}{c}\rho_{\mathrm{c}} 10^{15} \\
\mathrm{~g} / \mathrm{cm}^{3}\end{array}$ & $\begin{array}{c}R \\
\mathrm{~km}\end{array}$ & $\begin{array}{c}M \\
M_{\odot}\end{array}$ & $\begin{array}{c}\nu_{0} \\
\mathrm{kHz}\end{array}$ & $\begin{array}{c}\nu_{1} \\
\mathrm{kHz}\end{array}$ & $\begin{array}{c}\nu_{2} \\
\mathrm{kHz}\end{array}$ \\
\hline 2.800 & 9.998 & 2.201 & $0.39^{*}$ & 6.43 & 9.71 \\
2.700 & 10.059 & 2.201 & 0.45 & 6.50 & 9.79 \\
2.600 & 10.122 & 2.199 & 0.77 & 6.57 & 9.88 \\
2.500 & 10.193 & 2.197 & 1.01 & 6.63 & 9.92 \\
2.400 & 10.269 & 2.192 & 1.21 & 6.69 & 9.98 \\
2.200 & 10.428 & 2.176 & 1.58 & 6.83 & 10.08 \\
2.000 & 10.598 & 2.148 & 1.92 & 6.98 & 10.22 \\
1.800 & 10.789 & 2.098 & 2.25 & 7.12 & 10.35 \\
1.400 & 11.203 & 1.890 & 2.89 & 7.26 & 10.54 \\
1.000 & 11.572 & 1.410 & 3.37 & 7.01 & 10.07 \\
0.800 & 11.737 & 1.032 & 3.25 & 6.59 & 7.58 \\
0.700 & 11.884 & 0.826 & 3.01 & 5.88 & 6.54 \\
0.600 & 12.189 & 0.632 & 2.63 & 4.50 & 5.76 \\
\hline
\end{tabular}

Table A.18. Data for the polytropic EOS with $n=1$ and $\kappa=100 \mathrm{~km}^{2}$

\begin{tabular}{cccccc}
\hline $\begin{array}{c}\rho_{\mathrm{c}} 10^{15} \\
\mathrm{~g} / \mathrm{cm}^{3}\end{array}$ & $\begin{array}{c}R \\
\mathrm{~km}\end{array}$ & $\begin{array}{c}M \\
M_{\odot}\end{array}$ & $\begin{array}{c}\nu_{0} \\
\mathrm{kHz}\end{array}$ & $\begin{array}{c}\nu_{1} \\
\mathrm{kHz}\end{array}$ & $\begin{array}{c}\nu_{2} \\
\mathrm{kHz}\end{array}$ \\
\hline 5.700 & 7.518 & 1.351 & $0.618^{*}$ & 7.582 & 11.569 \\
5.650 & 7.535 & 1.351 & 0.180 & 7.576 & 11.556 \\
5.600 & 7.554 & 1.351 & 0.358 & 7.569 & 11.542 \\
5.500 & 7.590 & 1.351 & 0.569 & 7.557 & 11.520 \\
5.300 & 7.667 & 1.350 & 0.838 & 7.524 & 11.457 \\
5.000 & 7.787 & 1.348 & 1.129 & 7.475 & 11.365 \\
4.000 & 8.256 & 1.326 & 1.755 & 7.244 & 10.950 \\
3.000 & 8.862 & 1.266 & 2.141 & 6.871 & 10.319 \\
2.000 & 9.673 & 1.126 & 2.323 & 6.237 & 9.295 \\
1.500 & 10.19 & 0.998 & 2.302 & 5.737 & 8.513 \\
1.000 & 10.81 & 0.802 & 2.150 & 5.007 & 7.394 \\
\hline
\end{tabular}

Table A.19. Data for the polytropic EOS with $n=0.8$ and $\kappa=700 \mathrm{~km}^{2.5}$

\begin{tabular}{cccccc}
\hline $\begin{array}{c}\rho_{\mathrm{c}} 10^{15} \\
\mathrm{~g} / \mathrm{cm}^{3}\end{array}$ & $\begin{array}{c}R \\
\mathrm{~km}\end{array}$ & $\begin{array}{c}M \\
M_{\odot}\end{array}$ & $\begin{array}{c}\nu_{0} \\
\mathrm{kHz}\end{array}$ & $\begin{array}{c}\nu_{1} \\
\mathrm{kHz}\end{array}$ & $\begin{array}{c}\nu_{2} \\
\mathrm{kHz}\end{array}$ \\
\hline 4.800 & 7.832 & 1.609 & $0.630^{*}$ & 7.601 & 11.626 \\
4.750 & 7.853 & 1.609 & 0.281 & 7.602 & 11.624 \\
4.700 & 7.874 & 1.609 & 0.459 & 7.604 & 11.623 \\
4.600 & 7.917 & 1.609 & 0.690 & 7.606 & 11.618 \\
4.500 & 7.961 & 1.608 & 0.862 & 7.608 & 11.612 \\
4.300 & 8.053 & 1.606 & 1.132 & 7.609 & 11.596 \\
4.000 & 8.199 & 1.600 & 1.455 & 7.610 & 11.573 \\
3.500 & 8.470 & 1.579 & 1.868 & 7.577 & 11.477 \\
3.000 & 8.778 & 1.539 & 2.199 & 7.501 & 11.314 \\
2.500 & 9.126 & 1.468 & 2.464 & 7.361 & 11.051 \\
2.000 & 9.509 & 1.351 & 2.656 & 7.121 & 10.636 \\
1.500 & 9.908 & 1.161 & 2.741 & 6.711 & 9.969 \\
1.000 & 10.251 & 0.865 & 2.649 & 5.998 & 8.856 \\
\hline
\end{tabular}

Table A.20. Data for the polytropic EOS with $n=0.5$ and $\kappa=210^{5} \mathrm{~km}^{4}$

\begin{tabular}{cccccc}
\hline $\begin{array}{c}\rho_{\mathrm{c}} 10^{15} \\
\mathrm{~g} / \mathrm{cm}^{3}\end{array}$ & $\begin{array}{c}R \\
\mathrm{~km}\end{array}$ & $\begin{array}{c}M \\
M_{\odot}\end{array}$ & $\begin{array}{c}\nu_{0} \\
\mathrm{kHz}\end{array}$ & $\begin{array}{c}\nu_{1} \\
\mathrm{kHz}\end{array}$ & $\begin{array}{c}\nu_{2} \\
\mathrm{kHz}\end{array}$ \\
\hline 3.500 & 8.604 & 2.120 & $0.322^{*}$ & 7.308 & 11.220 \\
3.450 & 8.629 & 2.120 & 0.244 & 7.344 & 11.268 \\
3.400 & 8.655 & 2.120 & 0.620 & 7.402 & 11.347 \\
3.300 & 8.708 & 2.120 & 0.848 & 7.452 & 11.412 \\
3.200 & 8.763 & 2.118 & 1.140 & 7.548 & 11.542 \\
3.000 & 8.881 & 2.111 & 1.519 & 7.698 & 11.740 \\
2.600 & 9.140 & 2.075 & 2.151 & 8.005 & 12.139 \\
2.200 & 9.419 & 1.988 & 2.716 & 8.305 & 12.516 \\
1.800 & 9.672 & 1.809 & 3.235 & 8.555 & 12.804 \\
1.400 & 9.784 & 1.484 & 3.665 & 8.651 & 12.850 \\
1.200 & 9.713 & 1.252 & 3.792 & 8.551 & 12.653 \\
1.000 & 9.491 & 0.977 & 3.870 & 8.369 & 12.334 \\
0.800 & 9.045 & 0.678 & 3.810 & 7.962 & 11.690 \\
\hline
\end{tabular}

